\title{
Tailed radio galaxies as tracers of galaxy clusters. Serendipitous discoveries with the GMRT
}

\author{
S. Giacintucci ${ }^{1,2}$ and T. Venturi ${ }^{1}$ \\ 1 INAF - Istituto di Radioastronomia, via Gobetti 101, 40129, Bologna, Italy \\ e-mail: tventuri@ira.inaf.it \\ ${ }^{2}$ Harvard-Smithsonian Centre for Astrophysics, 60 Garden Street, Cambridge, MA 02138, USA
}

Received 1 June 2009 / Accepted 14 July 2009

\section{ABSTRACT}

\begin{abstract}
Aims. We report on the discovery of four new radio galaxies with tailed morphology. Tailed radio galaxies are generally found in rich environments, therefore their presence can be used as tracer of a cluster.

Methods. The radio galaxies were found in the fields of Giant Metrewave Radio Telescope (GMRT) observations carried out at $610 \mathrm{MHz}$ and $327 \mathrm{MHz}$ devoted to other studies. We inspected the literature and archives in the optical and X-ray bands to search for galaxy clusters or groups hosting them.

Results. All the tailed radio galaxies serendipitously found in the GMRT fields are located in rich environments. Two of them belong to the candidate cluster NCS J090232 +204358 , located at $z_{\text {phot }}=0.0746$; one belongs to the cluster MaxBCGJ 223.97317+22.15620 at $z_{\text {phot }}=0.2619$; finally we suggest that the fourth one is probing a galaxy cluster at $z=0.1177$, located behind A 262, and so far undetected in any band. Our results strengthen the relevance of high sensitivity and high resolution radio data in the detection of galaxy clusters at intermediate redshift.
\end{abstract}

Key words. radiation mechanisms: non-thermal - galaxies: clusters: general

\section{Distorted radio sources in clusters of galaxies}

Radio galaxies located in dense environments, such as galaxy clusters, often show complex and prominently distorted radio structures. A common morphology is represented by tailed radio galaxies, i.e., double sources with FR-I or FR-I/FR-II morphology (Fanaroff \& Riley 1974), whose jets and lobes are bent in $\mathrm{U}$ or $\mathrm{C}$ shapes. In wide-angle-tail radio galaxies (WAT, or C-shaped) the angle formed by the jets and lobes is usually very large (e.g. O'Donoghue et al. 1993; Feretti \& Venturi 2002 for a review). The prototype of this class is 3C 465 in A 2634 (Eilek et al. 1984). Sources whose jets and lobes form a small angle are referred to as narrow-angle-tail radio galaxies (NAT, or U-shaped; see Bliton et al. 1998; Feretti \& Venturi 2002 and references therein). The prototype of this class is NGC 1265 in the Perseus cluster (Wellington et al. 1973; O'Dea \& Owen 1986).

There is general consensus that the origin of the distorted radio morphologies lies in the interaction of the radio galaxy with respect to the dense surrounding intracluster medium (ICM), even though the details of such interaction are not fully disentangled. NAT sources are usually associated with galaxies moving at high velocity in the gravitational potential of the cluster (Miley 1980). The ram pressure exerted by the external medium is expected to be large enough to curve the jets and sweep the radio emitting plasma behind the rapidly moving galaxy. In agreement with this idea, the optical counterparts of narrow-angle-tails are not the bright dominant $\mathrm{D}$ or cD galaxies, usually nearly at rest in the cluster potential well, but rather galaxies with fainter optical magnitude and lower radio luminosity (Rudnick \& Owen 1976; Valentijn 1979). However, the host galaxies of NATs have, on average, velocities similar to those of typical cluster members, rather than moving with the high peculiar velocity expected in the ram pressure scenario. Therefore, it has been suggested that the jet bending might be a by-product, at least partially, of bulk motions in the ICM induced by cluster-subcluster mergers (Bliton et al. 1998).

The physical mechanism responsible for the distorsion of the jets in WATs is not completely understood either. Both ram pressure (Owen \& Rudnick 1976; Begelman et al. 1979) and buoyancy forces, active if the jet density is lower than the external gas density (Burns \& Balonek 1982), may play a role, the latter being dominant at larger distances from the cluster centre (Sakelliou et al. 1996). However, the very low peculiar velocities of their optical counterparts (Bird 1994; Pinkney et al. 2000) the cluster dominant $\mathrm{D}$ or cD ellipticals - is not consistent with the observed curvatures. The presence of large scale bulk flows in the ICM, induced by cluster or group mergers, is an appealing alternative for the jet bending (Pinkney et al. 1994; Roettiger et al. 1996; Gomez et al. 1997). Finally, a connection between the radio bending and gas sloshing in cluster cores has recently been suggested (e.g. A 2029; Clarke et al. 2004; Ascasibar \& Markevitch 2006).

The association between tailed radio galaxies and clusters can be used to trace the high density structures in the Universe. Indeed many previously unobserved clusters have been identified thanks to the detection of tailed sources (e.g. Fomalont \& Bridle 1978; Burns \& Owen 1979; and more recently Blanton et al. 2000, 2001 and 2003; Smolčić et al. 2007; Kantharia et al. 2009). In this paper we report on the discovery with the Giant Metrewave Radio Telescope (GMRT) of a number of distorted radio galaxies and on the successful search for the galaxy clusters in which they reside.

The paper is organised as follows: in Sect. 2 we present the images of the new tailed radio sources; in Sects. 3 and 4 we 
Table 1. Details of the GMRT observations.

\begin{tabular}{|c|c|c|c|c|c|c|c|}
\hline $\begin{array}{l}\text { Source name } \\
\text { (GMRT -) }\end{array}$ & $\begin{array}{l}\text { Pointing RA and Dec } \\
\left(\mathrm{h}, \mathrm{m}, \mathrm{s} \text { and }{ }^{\circ},,^{\prime},{ }^{\prime \prime}\right)\end{array}$ & $\begin{array}{l}d \\
\left({ }^{\prime}\right)\end{array}$ & $\begin{array}{c}v \\
(\mathrm{MHz})\end{array}$ & $\begin{array}{c}\Delta v \\
(\mathrm{MHz})\end{array}$ & $\begin{array}{l}\text { Obs. time } \\
(\min )\end{array}$ & $\begin{array}{c}\text { HPBW, PA } \\
\left(" x^{\prime \prime},{ }^{\circ}\right)\end{array}$ & $\begin{array}{c}\mathrm{rms} \\
\left(\mathrm{mJy} \mathrm{b}^{-1}\right)\end{array}$ \\
\hline $\mathrm{J} 0902+2044, \mathrm{~J} 0902+2045$ & 090045.9 & 27 & 610 & 32 & 80 & $6.5 \times 4.5,80$ & 60 \\
\hline $\mathrm{J} 1455+2209$ & 145715.2 & 22 & 610 & 32 & 100 & $6.1 \times 4.1,39$ & 60 \\
\hline J0154+3627 & 015250.0 & 32 & 327 & $16^{*}$ & 270 & $12.2 \times 8.7,87$ & 440 \\
\hline
\end{tabular}

* The observations were carried out using a total bandwidth of $32 \mathrm{MHz}$ (USB+LSB), but only the USB dataset was used for the analysis.

describe the optical identification procedure and the association with candidate clusters with photometric redshift; a brief summary and conclusions are reported in Sect. 5.

We adopt the $\Lambda$ CDM cosmology, with $H_{0}=$ $70 \mathrm{~km} \mathrm{~s}^{-1} \mathrm{Mpc}^{-1}, \Omega_{\mathrm{m}}=0.3$ and $\Omega_{\Lambda}=0.7$. The spectral index $\alpha$ is defined according to $\mathrm{S} \propto v^{-\alpha}$.

\section{Discovery of new radio galaxies}

In Venturi et al. $(2007,2008)$ we presented a large radio survey of a sample of galaxy clusters carried out with the GMRT at 610 $\mathrm{MHz}$ (the GMRT Radio-Halo Survey). During the analysis of those observations, we discovered three tailed radio galaxies in the wide-field images of two clusters of the sample. In particular, two of them, GMRT-J 0902+2044 and GMRT-J 0902+2045, are located at $\sim 27^{\prime}$ from the phase centre of the observation of Z 2089; the third tailed radio source, GMRT-J $1455+2209$, is located at $\sim 22^{\prime}$ from the pointing of the cluster Z 7160 .

Similarly, we found a diffuse and amorphous radio source, GMRT-J0154+3627, while imaging the $327 \mathrm{MHz}$ emission of the cluster A262 (archival GMRT observations), as part of a large observational project devoted to the study of the radio source feedback in groups and poor clusters of galaxies (Giacintucci et al. in prep.). The source is located at $\sim 32^{\prime}$ to the North-East of the antenna pointing of A 262.

Since the half power width of the primary beam of a GMRT antenna is $\sim 50^{\prime}$ at $610 \mathrm{MHz}$ and $\sim 90^{\prime}$ at $327 \mathrm{MHz}$, all the new radio sources lie well within the primary beam of the $Z 2089$, Z 7160 and A 262 fields.

\subsection{GMRT radio observations}

The details on the GMRT observations are summarised in Table 1, where we provide the radio source name; pointing coordinates of the observation (J2000); distance from the pointing; frequency, bandwidth and total lenght of the observations; half power beamwidth (HPBW) of the full array; rms level $(1 \sigma)$ measured in the region of the sources, prior to the primary beam correction. All the GMRT observations in Table 1 were obtained using both the upper and lower side bands of $16 \mathrm{MHz}$ each. The data were acquired in spectral line mode, with 128 channels/band and a spectral resolution of $125 \mathrm{kHz} / \mathrm{channel}$. The data reduction was carried out using the standard procedure (calibration, Fourier inversion, clean and restore) with the NRAO astronomical image processing system (AIPS) package. In order to reduce the size of the datasets and to minimize bandwidth smearing effects within the primary beam, after bandpass calibration the central channels of each observation were averaged to 6 channels of $\sim 2 \mathrm{MHz}$ each. After further careful editing in the averaged datasets, a number of phase-only self-calibration cycles and wide-field imaging were carried out for each band. The final images were produced by combining the USB and LSB self-calibrated datasets. Due to residual RFI in the LSB dataset of J 0154+3627 (A 262), the data combination led to images whose quality is worse than those obtained from the USB alone. For this reason only the USB dataset was used for the analysis presented in this paper. Residual amplitude calibration errors are $\leq 5 \%$. We refer to Venturi et al. $(2007,2008)$ and Giacintucci et al. (2008) for a complete description of the data reduction.

\subsection{The $610 \mathrm{MHz}$ images of the tailed radio galaxies}

In Figure 1 we show the $610 \mathrm{MHz}$ contours of the two tailed radio sources GMRT-J 0902+2044 and GMRT-J 0902+2045 (hereinafter J 0902+2044 and J 0902+2045 respectively), discovered in the field of Z2089 (Table 1). The two radio galaxies, both extending well beyond the optical size of the associated counterpart, are separated by $\sim 2.6^{\prime}$ in the plane of the sky. The contours are superposed to the red optical image from the Sloan Digital Sky Survey (SDSS; Data Release $7^{1}$ ). The radio image has been corrected for the primary beam attenuation of the GMRT at $610 \mathrm{MHz}$. In the region shown in Fig. 1 the sensitivity is $60 \mu \mathrm{Jy} \mathrm{b}^{-1}$, similar to the value achieved in the phase centre of the observations ( $45 \mu \mathrm{Jy} \mathrm{b}^{-1}$; Venturi et al. 2008). After the primary beam correction, the noise in the region of the radio galaxies increases to $150 \mu \mathrm{Jy} \mathrm{b}^{-1}$.

J 0902+2044 has a symmetric WAT morphology, with a central compact component and two bright jets marginally deflected to form an angle of $\sim 170^{\circ}$, at least out to $\sim 0.5^{\prime}$ from the central radio peak. Then the jets abruptly change direction by $\sim 90^{\circ}$, and merge into a single low surface brightness tail extending East of the source. A compression of the radio isophotes is visible in the North-Western edge of the jets, suggesting that the pressure necessary to bend the radio emission is coming from that direction.

The radio galaxy $\mathrm{J} 0902+2045$ is characterized by twin-jets, smoothly curved in a C-shape. The jets appear rather symmetric in morphology and extent, and can be traced out to $\sim 1.5^{\prime}$ from the central radio peak. The angle between the tails is $\sim 120^{\circ}$ out to approximately $30^{\prime \prime}$ from the centre. Then the jets undergo two more changes of direction, each with an angle of $\sim 120^{\circ}$; after the last bend, they lose collimation and gently curve toward the outer regions. From a morphological point of view, the very smooth and complete bending of J $0902+2045$ resembles that of NAT radio galaxies, and indeed the source is strongly reminiscent of the prototypical narrow-angle-tail source NGC 1265 in the Perseus cluster (e.g. O'Dea \& Owen 1986; see their Figs. 4 and 5); at the same time, radio sources similar to J 0902+2045 have also been classified in the literature as WAT (Smolčić et al. 2007).

The integrated flux density at $610 \mathrm{MHz}$ is $\mathrm{S}_{610 \mathrm{MHz}}=$ $258.2 \pm 12.9 \mathrm{mJy}$ for $\mathrm{J} 0902+2044$ and $\mathrm{S}_{610} \mathrm{MHz}=145.6 \pm 7.3$ mJy for J 0902+2045. Using the images from the NRAO VLA Sky Survey (NVSS; Condon et al. 1998), we measure a flux at $1.4 \mathrm{GHz}$ of $128.9 \pm 5.1 \mathrm{mJy}$ and $78.6 \pm 3.1 \mathrm{mJy}$, respectively. By comparison of these values with the $610 \mathrm{MHz}$ fluxes, we

\footnotetext{
1 wWW . sdss . org/dr7/
} 


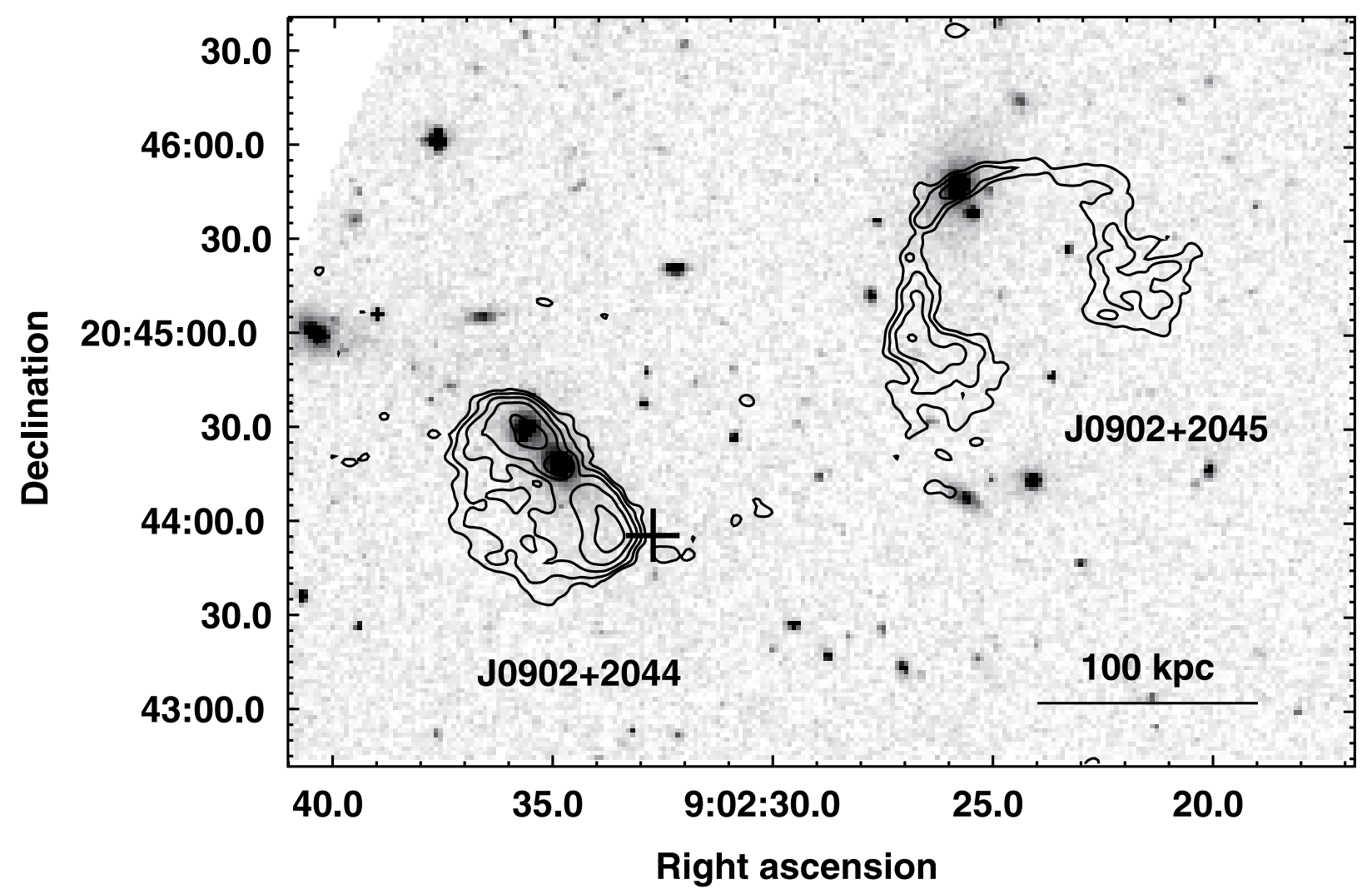

Fig. 1. GMRT $610 \mathrm{MHz}$ contours of the two tailed radio galaxies discovered at $\sim 27$ ' from the galaxy cluster Z2089. The image is corrected for the primary beam. The radio contours are overlaid on the red optical image from the SDSS. The resolution of the radio image is $6.5^{\prime \prime} \times 4.5^{\prime \prime}$, PA $80^{\circ}$. The lowest contour is $0.5 \mathrm{mJy} \mathrm{b}^{-1}$, and each contour increases by a factor of two. The cross indicates the centre of the candidate galaxy cluster NSC J090232+204358. The linear scale is $1^{\prime \prime}=1.56 \mathrm{kpc}$ (see Sect. 3).

Table 2. Radio and optical data of the tailed radio galaxies.

\begin{tabular}{|c|c|c|c|c|c|c|}
\hline $\begin{array}{l}\text { Radio name } \\
\text { (GMRT-) } \\
\text { Optical name } \\
\text { (SDSS-) }\end{array}$ & $\begin{array}{c}\mathrm{RA}_{\text {radio }} \\
(\mathrm{h}, \mathrm{m}, \mathrm{s}) \\
\mathrm{RA}_{\mathrm{opt}} \\
(\mathrm{h}, \mathrm{m}, \mathrm{s})\end{array}$ & $\begin{array}{l}\text { Dec } c_{\text {radio }} \\
\left({ }^{\circ},,^{\prime},{ }^{\prime}\right) \\
\text { Dec }_{\text {opt }} \\
\left({ }^{\circ},,^{\prime},{ }^{\prime}\right) \\
\end{array}$ & $\begin{array}{c}S_{610 \mathrm{MHz}} \\
(\mathrm{mJy}) \\
z_{\mathrm{spec}}\end{array}$ & $\begin{array}{c}S_{1.4 \mathrm{GHz}} \\
(\mathrm{mJy}) \\
M_{\mathrm{R}}\end{array}$ & $\begin{array}{c}\alpha_{0.6 \mathrm{GHz}}^{1.4 \mathrm{GHz}} \\
\log P_{1.4 \mathrm{GHz}} \\
\left(\mathrm{W} \mathrm{Hz}^{-1}\right)\end{array}$ & $\begin{array}{c}\text { Radio } \\
\text { morphology } \\
\text { LLS } \\
(\mathrm{kpc}) \\
\end{array}$ \\
\hline $\mathrm{J} 0902+2044$ & 090234.93 & +204417.9 & $258.2 \pm 12.9$ & $128.9 \pm 5.1$ & $0.83 \pm 0.11$ & WAT \\
\hline J $090234.90+204417.9$ & 090234.90 & +204418.0 & $0.0830 \pm 0.0002$ & -22.42 & 24.34 & 100 \\
\hline J $0902+2045$ & 090225.89 & +204546.6 & $145.6 \pm 7.3$ & $78.6 \pm 3.1$ & $0.74_{-0.11}^{+0.10}$ & NAT \\
\hline J 090225.86+204546.5 & 090225.87 & +204546.5 & $0.0820 \pm 0.0002$ & -22.21 & 24.11 & 300 \\
\hline J $1455+2209$ & 145553.51 & +220923.4 & $91.3 \pm 4.6$ & $49.1 \pm 2.0$ & $0.75_{-0.12}^{+0.10}$ & WAT \\
\hline J 145553.56+220922.3 & 145553.56 & +220922.3 & $0.2566 \pm 0.0002$ & -22.61 & 24.97 & 230 \\
\hline
\end{tabular}

obtained a spectral index $\alpha=0.83 \pm 0.11$ for the $\mathrm{J} 0902+2044$ and $\alpha=0.74_{-0.11}^{+0.10}$ for $\mathrm{J} 0902+2045$ (see Table 2).

Figure 2 shows the $610 \mathrm{MHz}$ image of the WAT GMRT-J 1455+2209 (hereinafter J 1455+2209), discovered at $\sim 22^{\prime}$ from Z7160 (Table 1), overlaid on the SDSS red optical frame. The sensitivity in this region is $60 \mu \mathrm{Jy} \mathrm{b}^{-1}$, and the noise level after the primary beam correction is $130 \mu \mathrm{Jy} \mathrm{b}^{-1}$. The source has a morphology similar to J 0902+2044 (Fig. 1), however no clear compact component is visible at the source centre, and some level of asymmetry is observed in the jet brightness. The flux density of $\mathrm{J} 1455+2209$ at $610 \mathrm{MHz}$ and $1.4 \mathrm{GHz}$ are $\mathrm{S}_{610 \mathrm{MHz}}=91.3 \pm 4.6 \mathrm{mJy}$ and $\mathrm{S}_{1.4 \mathrm{GHz}}=49.1 \pm 2.0 \mathrm{mJy}$ (from the NVSS), implying $\alpha=0.75_{-0.12}^{+0.10}$ (see Table 2).

The spectral index $\alpha$ calculated for the three tailed sources is in good agreement with the typical values of extended active radio galaxies. The radio information is summarised in Table 2.
For the optical properties provided in the table, we refer to Sect. 3 .

\subsection{The diffuse radio source J 0154+3627}

Figure 3 shows the $327 \mathrm{MHz}$ images of the diffuse radio source GMRT-J 0154+3627 (hereinafter J0154+3627), found in the periphery of A 262. In the literature this source is known as B2 0151+36, being part of the Second Bologna Catalog (B2) of radio sources (Colla et al. 1973), obtained using the Bologna Northern Cross telescope at $408 \mathrm{MHz}$. However, given the low resolution of that instrument $\left(3^{\prime}\right.$ in $\mathrm{RA}$, and $10^{\prime}$ in Dec), no morphological classification was provided at that time. The $327 \mathrm{MHz}$ full resolution image, shown in the left panel of Fig. 3 overlaid on the POSS-2 red optical plate, clearly reveals the presence of a bright and compact component at the source centre, 


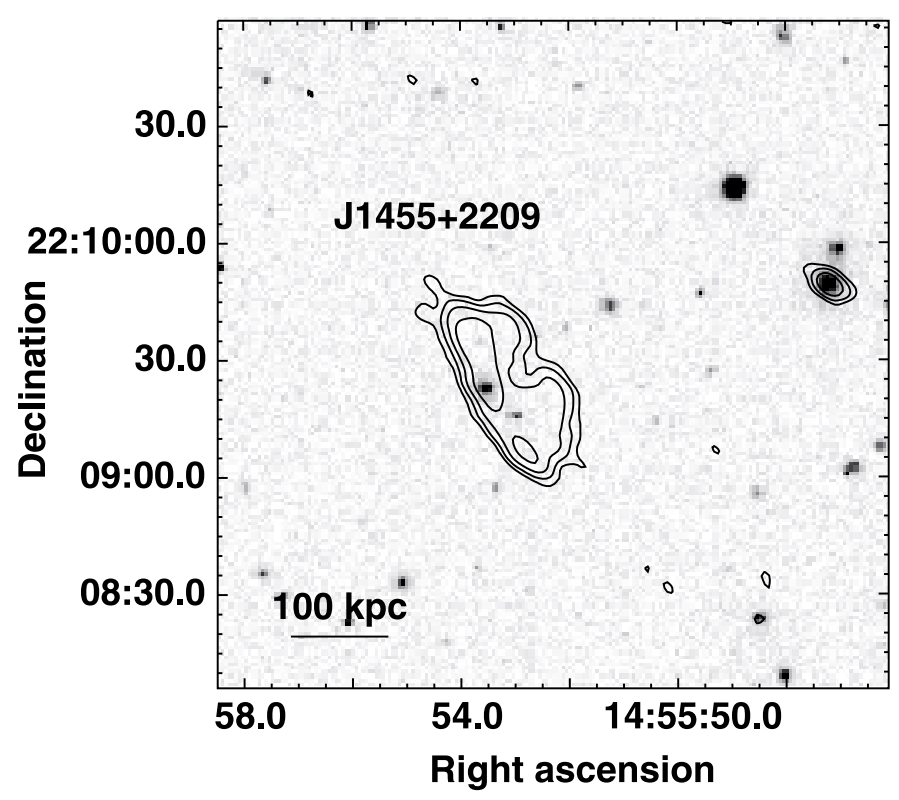

Fig. 2. GMRT $610 \mathrm{MHz}$ contours of the WAT discovered at $\sim 22^{\prime}$ from the galaxy cluster Z 7160. The image is corrected for the primary beam. The radio contours are overlaid on the red optical image from the SDSS. The resolution of the radio image is $6.1^{\prime \prime} \times 4.1^{\prime \prime}$, PA $39^{\circ}$. The lowest contour is $0.4 \mathrm{mJy} \mathrm{b}^{-1}$, and each contour increases by a factor of two. The centre of the galaxy cluster MaxBCG J223.97317+22.15620 is coincident with the elliptical galaxy associated with the WAT. The linear scale is $1^{\prime \prime}=3.99 \mathrm{kpc}$ (see Sect. 3).

two short symmetric jets (extended $\sim 1.5^{\prime}$ ), and a region of low brightness radio emission, with a rather amorphous morphology and a size of $\sim 2.5^{\prime} \times 3^{\prime}$. To better highlight the distribution of the low surface brightness emission, we produced a low resolution image at $327 \mathrm{MHz}$, shown in the right panel of Fig. 3. At this lower resolution $\left(28.8^{\prime \prime} \times 21.3^{\prime \prime}\right)$ the source reveals two faint radio tails, bent toward the same south-western direction. These tails can be well traced out to a distance of $\sim 3^{\prime}$ from the compact component. However, positive residuals of emission suggest that they might be more even extended than what is detected at the sensitivity level of the image.

We searched the radio archives for available observations of this source at different frequencies. We found two Very Large Array (VLA) observations at $1.4 \mathrm{GHz}$ and $4.9 \mathrm{GHz}$ (both in the C-array configuration; project $\mathrm{AC} 483$ ), that we re-analysed. In Fig. 4 we show the $4.9 \mathrm{GHz}$ contours $\left(\sim 5^{\prime \prime}\right.$ resolution) superposed to the grey scale image at $1.4 \mathrm{GHz}\left(\sim 16^{\prime \prime}\right.$ resolution). At $4.9 \mathrm{GHz}$ the extended emission is mostly elongated along the North-South axis, consistent with the inner part of the $327 \mathrm{MHz}$ image. The observed morphology at $1.4 \mathrm{GHz}$ is very similar to the images at $327 \mathrm{MHz}$ (Fig. 3). An elongated region of very low brightness emission is detected on the western side of the source, where the low resolution image at $327 \mathrm{MHz}$ reveals the existence of two radio tails.

The total flux density at $327 \mathrm{MHz}$, measured on the low resolution image in Fig. 3, is $620.5 \pm 31.0 \mathrm{mJy}$. From the images in Fig. 4, we obtained a flux of $234.4 \pm 9.4 \mathrm{mJy}$ at $1.4 \mathrm{GHz}$ and $132.8 \pm 5.3 \mathrm{mJy}$ at $4.9 \mathrm{GHz}$. The total spectral index is $\alpha=0.66_{-0.6}^{+0.7}$ between $327 \mathrm{MHz}$ and $1.4 \mathrm{GHz}$, and $\alpha=0.57_{-0.4}^{+0.3}$ between $327 \mathrm{MHz}$ and $4.9 \mathrm{GHz}$. The radio information is summarised in Table 3.

The compact component accounts for $\sim 3 \%$ only of the total flux at $327 \mathrm{MHz}$ (full resolution), while at higher frequency its contribution increases to $\sim 6 \%$ at $1.4 \mathrm{GHz}$ and $\sim 7 \%$ at $4.9 \mathrm{GHz}$.
Its spectral index is flat $(\alpha=0.2)$ over the whole frequency range, indicating that this component hosts the radio core of $\mathrm{J} 0154+3627$. The spectral index of the diffuse emission is $\alpha=$ 0.6 in the $327 \mathrm{MHz}-4.9 \mathrm{GHz}$ interval.

In Fig. 5 we show the integrated radio spectrum of the source between $327 \mathrm{MHz}$ and $4.9 \mathrm{GHz}$ (empty circles). The spectrum was obtained by combining the fluxes in Tab. 3, with the literature data provided by the NASA/IPAC Extragalactic Database (NED) for B2 $0151+36$. The spread in the flux values at 1.4 and $4.9 \mathrm{GHz}$ is most likely due to the different angular resolution of the observations. In the figure we also show the spectrum of the core (filled triangles) and of the diffuse component (filled circles).

The morphological classification of this radio galaxy is not straightforward. The image shown in the left panel of Fig. 3 is reminiscent of 3C 317, classified as a core-halo radio galaxy (Zhao et al. 1993), a class of sources typically found at the centres of cool core clusters (e.g. Baum \& O'Dea 1991; Sarazin et al. 1995; Mazzotta \& Giacintucci 2008). However, the diffuse emission in core-halo radio galaxies usually has a steep spectrum $(\alpha \geq 1)$, which is not observed here. Moreover, the western extension of the diffuse emission is suggestive of a tailed radio galaxy.

\section{Tailed radio galaxies as probes of the cluster environment}

We searched the literature to collect information about the environment of the new tailed radio galaxies presented in Sect. 2.2.

We found that $\mathrm{J} 0902+2044$ and $\mathrm{J} 0902+2045$ (Fig. 1) are located in the region of NSC J090232+204358 (hereinafter NSC J090+2043), a candidate galaxy cluster, on the basis of photometric redshifts, listed in the catalog of the Northern Sky Optical Cluster Survey (Gal et al. 2003). Its photometric redshift is $z_{\text {phot }}=0.075 \pm 0.033$, and its measured richness is $N_{\text {gal }}=39.2$ (Table 4).

The WAT J 1455+2209 (Fig. 2) is associated with the central galaxy of MaxBCG J223.97317+22.15620, classified as galaxy cluster by Koester et al. (2007) using the photometric information in the Sloan Digital Sky Survey. For this cluster Koester et al. provide $z_{\text {phot }}=0.262 \pm 0.010$ and $N_{\text {gal }}=18$. Table 4 summarizes the general properties of the two candidate galaxy clusters.

We inspected the optical images from the SDSS to identify the host galaxies of the three radio sources. The optical counterparts of J $0902+2044$ and $\mathrm{J} 0902+2045$ are two elliptical galaxies with spectroscopic redshift $z_{\mathrm{spec}}=0.083$ and $z_{\mathrm{spec}}=0.082$, respectively ${ }^{2}$. The agreement between these redshifts and the photometric redishift of the cluster, coupled with the tailed morphology of the two radio galaxies, confirms that NSC J090+2043 is the cluster hosting them.

The WAT J $1455+2209$ is associated with an elliptical at $z_{\text {spec }}=0.257^{3}$. Also in this case the galaxy redshift is consistent, within the errors, with the photometric redshift of the cluster MaxBCG J223.97317+22.15620 (Table 4).

The optical properties of the three tailed radio galaxies are summarised in Tab. 2, where we provide the optical name and coordinates, spectroscopic redshift, and absolute red magnitude $M_{\mathrm{R}}$. All the optical information is taken from the latest release of the SDSS (Data Release 7).

\footnotetext{
2 At these redshifts the linear scale is $1.56 \mathrm{kpc} /{ }^{\prime \prime}$

3 At this redshift the linear scale is $3.99 \mathrm{kpc} /$ "
} 

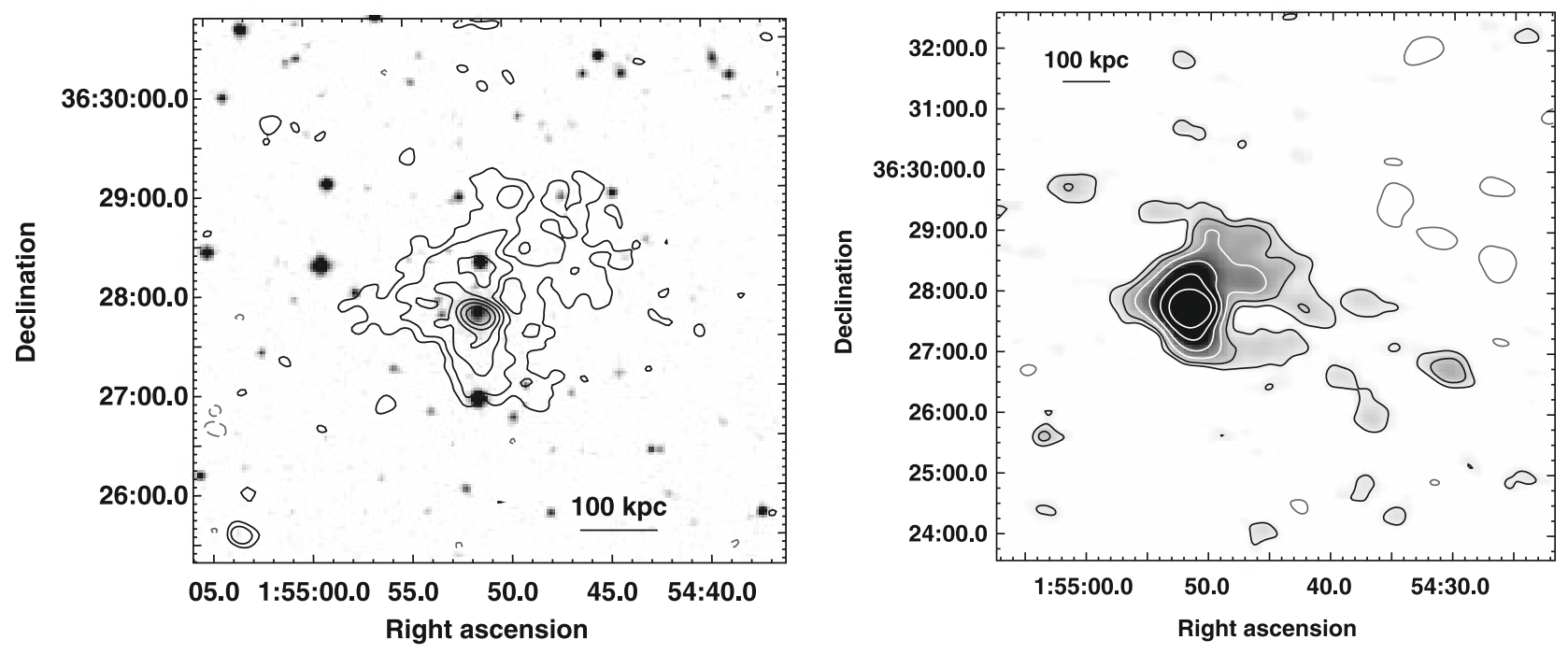

Fig. 3. Left: GMRT $327 \mathrm{MHz}$ full resolution contours of the diffuse radio source J0154+3627, discovered at $\sim 32$ ' from A 262 . The image is corrected for the primary beam attenuation. The radio contours are overlaid on the red optical image from the POSS-2. The resolution of the radio image is $12.2^{\prime \prime} \times 8.7^{\prime \prime}$, PA $87^{\circ}$. The lowest contour is $1.6 \mathrm{mJy} \mathrm{b}^{-1}$, and each contour increases by a factor of two. Right: GMRT $327 \mathrm{MHz}$ low resolution image (contours and grey scale) of $\mathrm{J} 0154+3627$. The resolution is $28.8^{\prime \prime} \times 21.3^{\prime \prime}$, PA -88 . Contours are spaced by a factor 2 , starting from $\pm 3 \sigma\left(1 \sigma=600 \mathrm{mJy} \mathrm{b}^{-1}\right)$. For this source the scale is $2.127 \mathrm{kpc} /{ }^{\prime \prime}$ (see Sect. 4).

Table 3. Radio and optical data of the candidate NAT J 0154+3627.

\begin{tabular}{|c|c|c|c|c|c|c|c|}
\hline $\begin{array}{l}\text { Radio name } \\
\text { (GMRT-) }\end{array}$ & $\begin{array}{l}\mathrm{RA}_{\text {radio }} \\
(\mathrm{h}, \mathrm{m}, \mathrm{s})\end{array}$ & $\begin{array}{l}\text { Dec }_{\text {radio }} \\
\left({ }^{\circ},{ }^{\prime},\right)\end{array}$ & $\begin{array}{c}S_{327 \mathrm{MHz}} \\
(\mathrm{mJy})\end{array}$ & $\begin{array}{c}S_{1.4 \mathrm{GHz}} \\
(\mathrm{mJy})\end{array}$ & $\begin{array}{c}S_{4.9 \mathrm{GHz}} \\
(\mathrm{mJy})\end{array}$ & $\begin{array}{c}\alpha_{0.3 \mathrm{GHz}}^{1.4 \mathrm{GHz}} \\
\left(\mathrm{W} \mathrm{Hz}^{-1}\right)\end{array}$ & $\log P_{1.4 \mathrm{GHz}}$ \\
\hline $\begin{array}{l}\text { Optical name } \\
\text { (NFP-) }\end{array}$ & $\begin{array}{c}\mathrm{RA}_{\mathrm{opt}} \\
(\mathrm{h}, \mathrm{m}, \mathrm{s})\end{array}$ & $\begin{array}{l}\mathrm{Dec}_{\mathrm{opt}} \\
\left({ }^{\circ},{ }^{\prime \prime}\right)\end{array}$ & $z_{\text {spec }}$ & $r$ & $M_{\mathrm{R}}$ & $\alpha_{0.3 \mathrm{GHz}}^{4.9 \mathrm{GHz}}$ & $\begin{array}{l}\text { LLS } \\
\mathrm{kpc}\end{array}$ \\
\hline J $0154+3627$ & 015451.53 & +362746.2 & $620.5 \pm 31.0$ & $234.4 \pm 9.4$ & $132.8 \pm 5.3$ & $0.66_{-06}^{+0.1}$ & 24.93 \\
\hline $\mathrm{J} 015451.5+362747$ & 090234.92 & +204418.0 & $0.1177 \pm 0.0002$ & 15.96 & -22.73 & $0.57_{-04}^{+0.3}$ & 340 \\
\hline
\end{tabular}

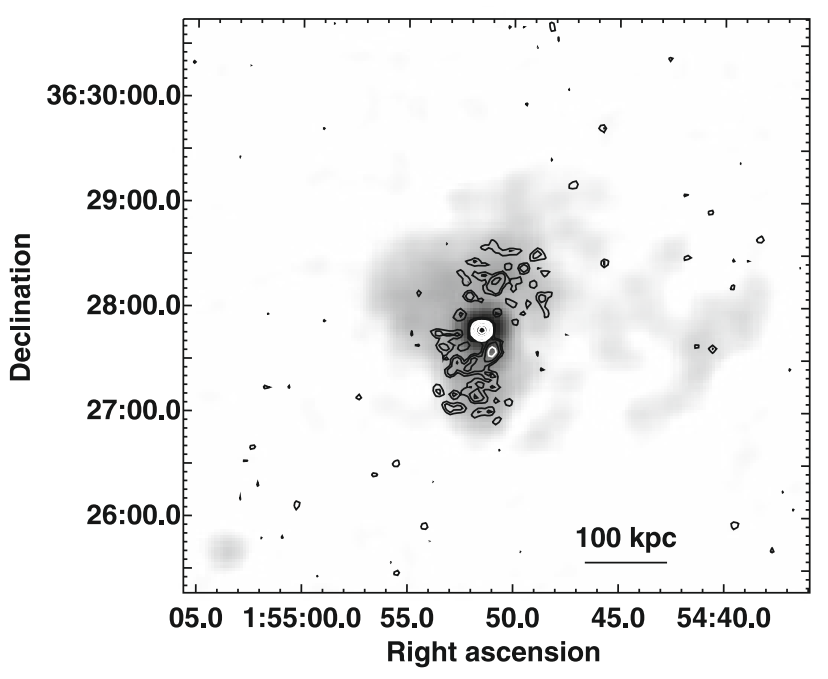

Fig. 4. VLA $4.7 \mathrm{GHz}$ contours on the $1.4 \mathrm{GHz}$ grey scale image of $\mathrm{J} 0154+3627$. The resolution is $4.9^{\prime \prime} \times 4.2^{\prime \prime}$, PA -80 and $15.8^{\prime \prime} \times$ 13.4", $\mathrm{PA}-74$. Contours are spaced by a factor 2 , starting from $\pm 3 \sigma$ $\left(1 \sigma=45 \mu \mathrm{Jy} \mathrm{b}^{-1}\right.$ and $\left.1 \sigma=50 \mu \mathrm{Jy} \mathrm{b}^{-1}\right)$. For this source the scale is $2.127 \mathrm{kpc} /{ }^{\prime \prime}$.

Using the redshift of the optical counterparts and the NVSS flux density in Table 2, we calculated the radio power at $1.4 \mathrm{GHz}$.
The WAT and the NAT in NSC J090+2043 have $P_{1.4 \mathrm{GHz}}=$ $2.2 \times 10^{24} \mathrm{~W} \mathrm{~Hz}^{-1}$ and $1.3 \times 10^{24} \mathrm{~W} \mathrm{~Hz}^{-1}$, respectively. The radio power of the WAT J $1455+2209$ is $P_{1.4} \mathrm{GHz}=9.3 \times 10^{24} \mathrm{~W} \mathrm{~Hz}^{-1}$ (Table 2). The combination of these radio powers and the optical magnitude of the associated galaxies, in the range $M_{\mathrm{R}}=$ $-22.21 \div-22.61$ (see Table 2) places them in the transition region between FRI and FRII radio galaxies in the $M_{\mathrm{R}}-\log P_{1.4 \mathrm{GHz}}$ diagram (Owen \& Ledlow 1994).

The projected linear sizes of these three radio galaxies, in the range 100-300 kpc (see Table 2), are consistent with what is typically found for tailed radio galaxies in clusters, whose sizes range over a wide interval, from tens to hundreds of $\mathrm{kpc}$.

We inspected the X-ray images from the ROSAT All Sky Survey (RASS) both for NCS J 0902+2043 and MaxBCG J 223.97317+22.15620. No X-ray counterpart is detected in both cases. Such lack of detection may be due to a number of reason. On one side, the number of optical galaxies provided in the literature (Table 4) suggests that they are poor clusters, and the lack of X-ray information might be due to the faint X-ray emission typical of these environments; on the other side, at the redshift of MaxBCG J 223.97317+22.15620 the cluster X-ray emission might be below the sensitivity limit of the RASS. We note, for instance, that the rich and X-ray luminous $\left(L_{\mathrm{X}}=9.25 \times 10^{44} \mathrm{erg} \mathrm{s}^{-1}\right)$ merging cluster RXCJ 2003.52323 , located at $z=0.3171$, was barely detected on the RASS, and deep pointed Chandra observations were necessary for a radio/X-ray analysis (Giacintucci et al. 2009). 
Table 4. General properties of the candidate galaxy clusters.

\begin{tabular}{ccccc}
\hline \hline Cluster name & $\begin{array}{c}\text { RA } \\
(\mathrm{h}, \mathrm{m}, \mathrm{s})\end{array}$ & $\begin{array}{c}\text { Dec } \\
\left({ }^{\circ},{ }^{\prime},{ }^{\prime \prime}\right)\end{array}$ & $z_{\text {phot }}$ & $N_{\text {gal }}$ \\
\hline NSC J090232+204358 & 090232.7 & +204357 & $0.075 \pm 0.033$ & 39.2 \\
MaxBCG J223.97317+22.15620 & 145553.5 & +220922 & $0.262 \pm 0.010$ & 18 \\
\hline
\end{tabular}

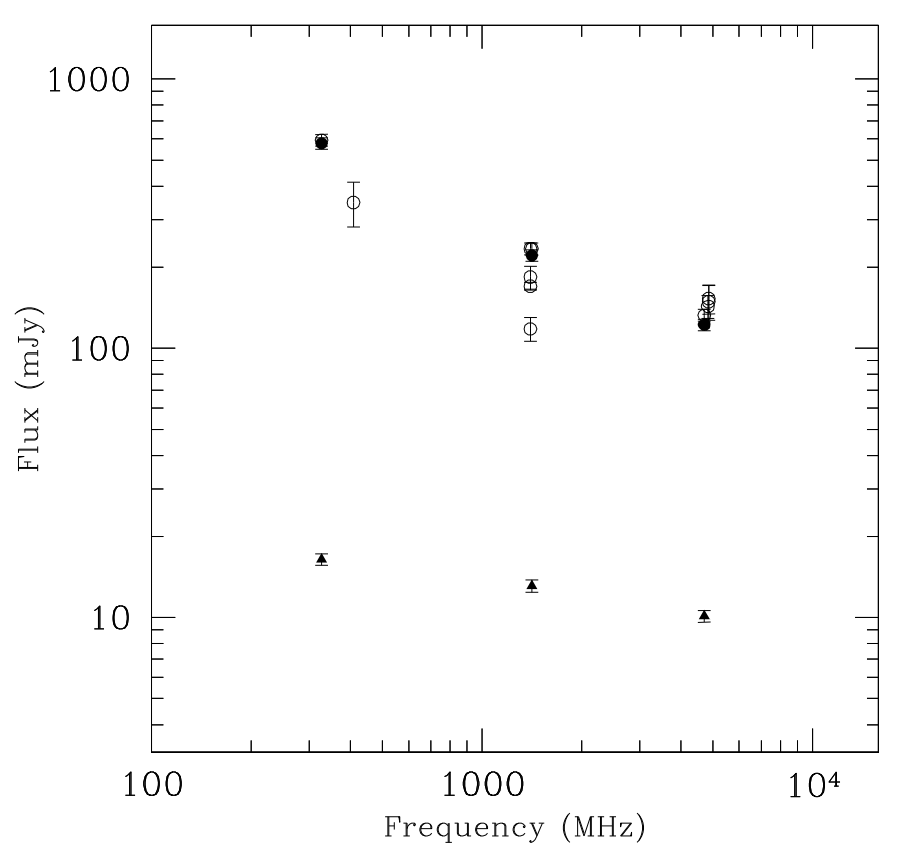

Fig. 5. Radio spectrum of the candidate tailed radio galaxy J0154+3627 between $327 \mathrm{MHz}$ and $4.9 \mathrm{GHz}$ (empty circles). The spectrum of the core and extended emission are shown as filled triangles and filled circles, respectively.

\section{A galaxy cluster behind A 262?}

As clear from Fig. 3 (left), the radio core of the candidate tailed source $\mathrm{J} 0154+3627$ is coincident with a bright optical galaxy on the POSS-2 image. Its projected distance from the centre of the galaxy cluster A 262 is $\sim 32^{\prime}$, which corresponds to $\sim 600 \mathrm{kpc}$ at the redshift of A $262(z=0.0163)$. However, its spectroscopic redshift, $z_{\text {spec }}=0.118^{4}$ (Smith et al. 2004), rules out the membership to A 262 .

Using the flux density measured on the NVSS, and assuming a redshift of 0.1177 , we calculated the radio power of the source at $1.4 \mathrm{GHz}$. We obtained $P_{1.4 \mathrm{GHz}}=8.5 \times 10^{24} \mathrm{~W} \mathrm{~Hz}^{-1}$. Also this radio galaxy is located in the FRI/FRII transition region in the $M_{\mathrm{R}}-\log P_{1.4 \mathrm{GHz}}$ plot. Note that this galaxy is the most luminous among those presented in this paper, even though it is not as bright as the brightest central dominant galaxies in clusters $\left(M_{\mathrm{R}} \sim-23\right.$, Owen \& Ledlow 1994).

It is difficult to provide an unambiguous morphological classification for $\mathrm{J} 0154+3627$, since projection effects are likely to play a role. The two short jets visible in Fig. 3, extending beyond the optical galaxy, and the asymmetry of the diffuse emission (extended westwards) lead us to think that we are dealing with a tailed source rather than with a core-halo radio galaxy. The bright optical magnitude of the associated galaxy and the radio power would be consistent with a centrally located WAT, but the possibility that we are dealing with a NAT radio galaxy

${ }^{4}$ At this redshift the linear scale is $2.13 \mathrm{kpc} / "$. cannot be ruled out. In any case, we can safely conclude that $\mathrm{J} 0154+3627$ is a tailed radio galaxy, most likely associated with a cluster at $z=0.1177$ located behind A 262 .

We checked on the X-ray archives in search for information in this band. J0154+3627 is located outside the field of view of all the pointed observations of A 262, and the only available image for this source is provided by the RASS. Figure 6 shows the smoothed RASS image in the $0.5-2.1 \mathrm{keV}$ band of A 262 and the region of J 0154+3627, with the GMRT contours at $327 \mathrm{MHz}$ overlaid. The image reveals a region of diffuse emission spatially coincident with the radio galaxy. Its projected distance from the centre of A 262 is $\sim 600 \mathrm{kpc}$, and thus we cannot rule out the possibility that such emission is a clump of gas or an infalling group at the outskirts of the cluster, rather than emission coming from a background cluster (or group). Pointed Xray observations would be useful to investigate the morphology of this emission and its possible connection to the radio galaxy J $0154+3627$.

\section{Summary and conclusions}

In this paper we report on the serendipitous discovery of four radio galaxies, whose tailed radio morphology is suggestive of a cluster environment. Such discovery was possible thanks to the high sensitivity and wide field of view of the GMRT at $610 \mathrm{MHz}$ (Venturi et al. 2007, 2008) and at $327 \mathrm{MHz}$.

We searched for the optical counterparts of the new tailed radio galaxies, and found that J 0902+2044 and J 0902+2045, respectively a WAT and a NAT, are located in the candidate galaxy cluster NCS J0902+2043 with photometric redshift 0.075. Our radio images thus confirm that NCS J0902+2043 is indeed a galaxy cluster, whose redshift is consistent with the radio power derived for the two radio galaxies.

Similarly, we confirm that MaxBCG J223.97317+22.15620 $\left(z_{\text {phot }}=0.262\right)$ is a galaxy cluster, which hosts the central WAT $\mathrm{J} 1455+2209$.

The optical counterpart of the candidate tailed source $\mathrm{J} 0154+3627$ is a galaxy at redshift $z=0.118$. This rules out the possibility that the radio galaxy is associated with A 262 $(z=0.016)$. The observational properties of the radio source, i.e., morphology, spectrum, radio power and size, suggest that it is member of a galaxy cluster, which is so far undetected in the optical band. Inspection of the X-ray RASS image reveals a clump of diffuse emission coincident with the radio galaxy. However, the available X-ray data do not allow us to tell whether such emission is related to the environment of $\mathrm{J} 0154+3627$ $(z=0.118)$, or to the closer A $262(z=0.016)$.

Our findings confirm that distorted radio galaxies can be used as tracers of galaxy clusters. The low observing frequencies available with the GMRT (few hundred of $\mathrm{MHz}$ ), coupled with its large field of view (primary beam $\sim 1$ to 2.5 degrees going from $610 \mathrm{MHz}$ to $240 \mathrm{MHz}$ ), very good sensitivity (from few tens to few hundreds of $\mu \mathrm{Jy}$ going from $610 \mathrm{MHz}$ to $240 \mathrm{MHz}$ ) and angular resolution (of the order of $5^{\prime \prime}$ and $15^{\prime \prime}$ respectively at $610 \mathrm{MHz}$ and $240 \mathrm{MHz}$ ) are an ideal combination for the discovery of galaxy clusters through tailed radio galaxies, not only 


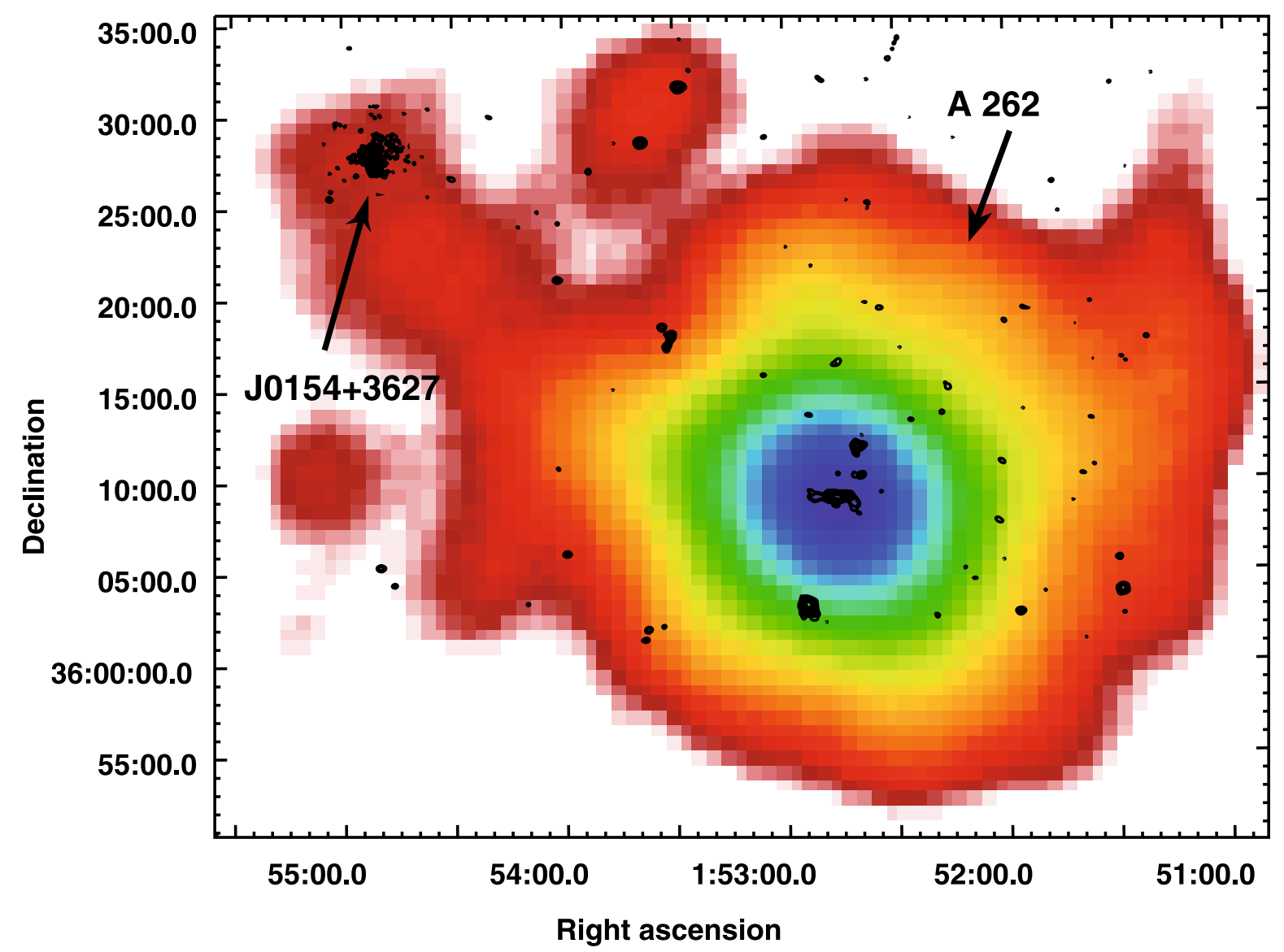

Fig. 6. GMRT $327 \mathrm{MHz}$ contours, overlaid on the RASS image in the $0.5-2.1 \mathrm{keV}$ band of the A 262 region. The resolution of the radio image is $19.5^{\prime \prime} \times 15.2^{\prime \prime}, \mathrm{PA}-86^{\circ}$. The lowest contour is at the $5 \sigma$ level $=2.5 \mathrm{mJy} \mathrm{b}^{-1}$, and each contours increases by actor of two. The RASS image has been smoothed with a gaussian with $\sigma=6^{\prime}$.

in the Local Universe but also at intermediate redshifts. We point out that the sensitivity of the REFLEX survey (Böhringer et al. 2004) does not allow to detect any cluster with X-ray luminosity $L_{\mathrm{X}} \leq 5 \times 10^{44} \mathrm{erg} \mathrm{s}^{-1}$ beyond $z \sim 0.1$, therefore the morphological properties of radio galaxies are the only observational signature to confirm the existence of gravitationally bound systems above such redshifts. Such result is relevant not only in itself, but also in the light of studies of the evolution of the radio galaxy population in clusters of galaxies.

Acknowledgements. We thank Prof. D. Dallacasa for insightful discussion and careful reading of the manuscript and Dr. F. Gastaldello for discussion and help in handling the X-ray data archives. We thank the staff of the GMRT for their help during the observations. GMRT is run by the National Centre for Radio Astrophysics of the Tata Institute of Fundamental Research. We acknowledge financial contribution from the Italian Ministry of Foreign Affairs, from MIUR grants PRIN2004, PRIN2005 and 2006, from PRIN-INAF2005 and from contract ASI-INAF I/023/05/01.

\section{References}

Ascasibar, Y., \& Markevitch, M. 2006, ApJ, 650, 102 Baum, S. A., \& O’Dea, C. P. 1991, MNRAS, 250, 737 Begelman, M. C., Rees, M. J., \& Blandford, R. D. 1979, Nature, 279, 770 Bird, C. M. 1994, AJ, 107, 1637

Blanton, E. L., Gregg, M. H., Helfand, D. J., et al. 2000, ApJ, 531, 118 Blanton, E. L., Gregg, M. H., Helfand, D. J., et al. 2001, AJ, 121, 2915 Blanton, E. L., Gregg, M. H., Helfand, D. J., et al. 2003, AJ, 125, 1635 Bliton, M., Rizza, E., Burns, J. O., et al. 1998, MNRAS, 301, 609 Böhringer, H., Schuecker, P., Guzzo, L., et al. 2004, A\&A, 425, 367 Burns, J. O., \& Owen, F. N. 1979, AJ, 84, 1478
Burns, J. O., \& Balonek, T. J. 1982, ApJ, 263, 546

Clarke, T. E., Blanton, E. L., \& Sarazin, C. L. 2004, ApJ, 616, 178 Colla, G., Fanti, C., Fanti, R., et al. 1973, A\&AS, 11, 291

Condon, J. J., Cotton, W. D., Greisen, E. W., et al. 1998, AJ, 115, 1693 Eilek, J. A., Burns, J. O., O’Dea, C. P., et al. 1984, ApJ, 278, 37 Gal, R. R., de Carvalho, R. R., Lopes, P. A. A., et al. 2003, ApJ, 125, 2064 Fanaroff, B. L., \& Riley, J. M. 1974, MNRAS, 167, 31

Feretti, L., \& Venturi, T. 2002, in Merging Processes in Galaxy Clusters, ed. L.

Feretti, I. M. Gioia, \& G. Giovannini, ASSL, 272, 163

Fomalont, E. B., \& Bridle, A. H. 1978, ApJ, 223, 9

Giacintucci, S., Venturi, T., Macario, G., et al. 2008, A\&A, 486, 347 Giacintucci, S., Venturi, T., Brunetti, G., et al. 2009, A\&A, 505, 45

Gomez, P. L., Pinkney, J., Burns, J. O., et al. 1997, ApJ, 474, 580

Kantharia, N. G., Das, M., \& Gopal-Krishna, arXiv: 0903.3815

Koester, B. P., McKay, T. A., Annis, J., et al. 2007, ApJ, 660, 239

Mazzotta, P., \& Giacintucci, S. 2008, ApJ, 675, 9

Miley, G. 1980, ARA\&A, 18, 165

O'Dea, C. P., \& Owen, F. N. 1986, ApJ, 301, 841

O'Donoghue, A. A., Eilek, J. A., \& Owen, F. N. 1993, ApJ, 408, 4280

Owen, F. N., \& Rudnick, L. 1976, ApJ, 205, 1

Owen, F. N., \& Ledlow, M. J. 1994, ASPC, 54, 319

Pinkney, J., Burns, J. O., \& Hill, J. M. 1994, AJ, 108, 2031

Pinkney, J., Burns, J. O., Ledlow, M. J., et al. 2000, AJ, 120, 2269

Roettiger, K., Burns, J. O., \& Loken, C. 1996, ApJ, 473, 651

Rudnick, L., \& Owen, F. N. 1976, AJ, 203, L107

Smith, R. J., Hudson, M. J., Nelan, J. E., et al. 2004, AJ, 128, 1558 S

Sakelliou, I., Merrifield, M. R., \& McHardy, I. M. 1996, MNRAS, 283, 673

Sarazin, C. L., Baum, S. A., \& O’Dea, C. P. 1995, ApJ, 451, 125

Smolčić, V., Schinnerer, E., Finoguenov, A., et al. 2007, ApJS, 172, $295 \mathrm{~S}$

Valentijn, E. A. 1979, A\&A, 78, 367

Venturi, T., Giacintucci, S., Brunetti, G., et al. 2007, A\&A, 463, 937

Venturi, T., Giacintucci, S., Dallacasa, D., et al. 2008, A\&A, 484, 327

Wellington, K., Miley, G., \& van der Laan, H. 1973, Nature, 244, 502 Zhao, J.-H., Sumi, D. M., Burns, J. O., et al. 1993, ApJ, 416, 51 